\title{
Reduced value-driven attentional capture among children with ADHD compared to typically developing controls
}

\author{
Anthony W. Sali ${ }^{1}$, Brian A. Anderson ${ }^{2}$, Steven Yantis ${ }^{3}$, Stewart H. Mostofsky ${ }^{4,5,6}$, and Keri \\ S. Rosch $^{4,5,7}$ \\ ${ }^{1}$ Center for Cognitive Neuroscience, Duke University, Durham NC, ORCID: 0000-0003-2297-7923 \\ 2Department of Psychology, Texas A\&M University, College Station TX \\ ${ }^{3}$ Department of Psychological and Brain Sciences, Johns Hopkins University, Baltimore, MD \\ ${ }^{4}$ Center for Neurodevelopmental and Imaging Research, Kennedy Krieger Institute, Baltimore, \\ $\mathrm{MD}$ \\ ${ }^{5}$ Departments of Psychiatry and Behavioral Sciences, Johns Hopkins University School of \\ Medicine, Baltimore, MD \\ ${ }^{6}$ Department of Neurology, Johns Hopkins University School of Medicine, Baltimore, MD \\ ${ }^{7}$ Neuropsychology Department, Kennedy Krieger Institute, Baltimore, MD
}

\section{Abstract}

\begin{abstract}
The current study examined whether children with ADHD were more distracted by a stimulus previously associated with reward, but currently goal-irrelevant, than their typically-developing peers. In addition, we also probed the associated cognitive and motivational mechanisms by examining correlations with other behavioral tasks. Participants included 8-12 year-old children with $\operatorname{ADHD}(n=30)$ and typically developing controls $(n=26)$. Children were instructed to visually search for color-defined targets and received monetary rewards for accurate responses. In a subsequent search task in which color was explicitly irrelevant, we manipulated whether a distractor item appeared in a previously reward-associated color. We examined whether children responded more slowly on trials with the previously-rewarded distractor present compared to trials without this distractor, a phenomenon referred to as value-driven attentional capture (VDAC), and whether children with and without ADHD differed in the extent to which they displayed VDAC. Correlations among working memory performance, immediate reward preference (delay discounting) and attentional capture were also examined. Children with ADHD were significantly less affected by the presence of the previously rewarded distractor than were control participants. Within the ADHD group, greater value-driven attentional capture was associated with poorer
\end{abstract}

Please direct correspondence to: Anthony W. Sali, Center for Cognitive Neuroscience, Duke University, anthony.sali@ duke.edu, 919-684-1034.

Conflict of Interest: The authors declare that they have no conflict of interest.

Ethical approval: All procedures performed in studies involving human participants were in accordance with the ethical standards of the institutional and/or national research committee and with the 1964 Helsinki declaration and its later amendments or comparable ethical standards.

Informed consent: Informed consent was obtained from all individual participants included in the study. 
working memory. Although both ADHD and control participants were initially distracted by previously reward-associated stimuli, the magnitude of distraction was larger and persisted longer among control participants.

\section{Keywords}

ADHD; attention; reward; distraction; reinforcement learning

\section{Introduction}

ADHD is the most commonly diagnosed mental health disorder in childhood involving persisting and impairing developmentally inappropriate levels of inattention or distractibility, hyperactivity, and impulsivity (American Psychiatric Association, 2013; Visser et al., 2014). Difficulties with attention regulation and distractibility are present in most individuals with ADHD and persist throughout development (Willcutt et al., 2012). Despite the centrality of distractibility to ADHD phenomenology and the empirical and theoretical emphasis on atypical motivation in ADHD, the extent to which valuable or reward-related stimuli are more distracting in children with ADHD has not yet been examined. The value-driven attentional capture (VDAC) paradigm (Anderson, Laurent, \& Yantis, 2011a, 2011b; Anderson \& Yantis, 2013; Yantis, Anderson, Wampler, \& Laurent, 2012), based in a cognitive neuroscience framework, allows us to directly examine the extent to which children with ADHD are distracted by previously reward-related stimuli relative to typically developing controls. The purpose of this study is to clarify the mechanisms contributing to distractibility in ADHD by translationally applying advances from cognitive neuroscience using a well-validated measure of attentional capture by valuable stimuli.

The deployment of attention has traditionally been thought to involve two inter-related modes: 1) a voluntary, goal-directed mode, in which attention is guided by contextually appropriate goals and intentions, and 2) an involuntary, stimulus-driven mode, in which attention is captured by physically salient stimuli (Theeuwes, 1992, 1994, 2010). Thus, the likelihood that a stimulus will be attended, referred to as the stimulus's attentional priority, reflects both the stimulus's physical properties as well as the moment-by-moment goals of the observer (Folk, Leber, \& Egeth, 2002; Folk, Remington, \& Johnston, 1992; Wolfe, Cave, $\&$ Franzel, 1989). However, children with ADHD do not differ from their age-matched controls in the degree to which the presence of a highly physically salient distractor induces oculomotor capture in visual search (Van der Stigchel et al., 2007). ADHD-associated deficits in attentional control are therefore unlikely to arise simply from an inability to suppress attentional orienting toward physically salient stimuli. Instead, ADHD may be associated with greater attentional orienting toward valuable (i.e., reward-related) stimuli.

Recent work has demonstrated that the brain is optimized to learn about perceptual stimuli that signal the potential for procuring reward. Accordingly, rewarding stimuli can exert a powerful influence on attentional deployment (Sali, Anderson, \& Courtney, 2016; Seitz, Kim, \& Watanabe, 2009; Shuler \& Bear, 2006). In fact, many studies have shown that reward facilitates voluntary attention to task-relevant stimuli, and that attentional 
performance is strongly influenced by reward-based strategies (Della Libera \& Chelazzi, 2006, 2009; Engelmann \& Pessoa, 2007; Hickey, Chelazzi, \& Theeuwes, 2010a, 2010b; Kiss, Driver, \& Eimer, 2009; Kristjansson, Sigurjonsdottir, \& Driver, 2010; Navalpakkam, Koch, \& Perona, 2009; Peck, Jangraw, Suzuki, Efem, \& Gottlieb, 2009; Pessoa \& Engelmann, 2010; Raymond \& O'Brien, 2009). However, involuntary attentional capture by rewarding stimuli can be maladaptive when it conflicts with contextually appropriate goals, i.e., when more immediately rewarding stimuli serve to distract a person from achieving a longer-term goal. For example, if a child is supposed to attend to their teacher but their attention is captured by more attractive and rewarding stimuli such as friends playing outside or thoughts of videogames, they may miss important information presented in class.

Similarly, stimuli that have come to be associated with reward have been shown to cause significant and persistent distraction, suggesting that there exists an involuntary mechanism of attentional selection that is uniquely value-driven (Anderson et al., 2011a, 2011b; Anderson \& Yantis, 2012, 2013). Further, there is some evidence that greater VDAC correlates with greater self-reported impulsivity and poorer working memory performance among healthy college students (Anderson et al., 2011b), and is associated with impulsive non-planning behaviors in HIV+ patients (Anderson, Kronemer, Rilee, Sacktor, \& Marvel, 2016). Thus, there is good reason to suspect greater VDAC among individuals with ADHD.

Individuals with ADHD may differ from typically developing children in their susceptibility to VDAC due to altered reward sensitivity. Research over the past few decades has shown that children with ADHD respond differently to reward both in terms of their behavior and physiology (see reviews by Luman, Oosterlaan, \& Sergeant, 2005; Luman, Tripp, \& Scheres, 2010). For example, studies have shown that performance-based rewards improve cognitive task performance among children with ADHD (e.g., Bubnik, Hawk, Pelham, Waxmonsky, \& Rosch, 2015; Epstein et al., 2011; Rosch et al., 2016; Rosch \& Hawk, 2013; Shiels et al., 2008; Strand et al., 2012) and that individuals with ADHD tend to show less activation in the striatum, a brain region central to reward processing, in response to cues predicting reward (see review by Plichta \& Scheres, 2014). However, further research is necessary to elucidate the nature of this atypical response to reward given the breadth of this topic and the numerous ways in which reward sensitivity can be defined and measured. To our knowledge, no previous studies have examined the extent to which individuals with ADHD are distracted by rewarding stimuli as reflected in the VDAC paradigm.

There are also few studies examining the relationship between cognitive and motivational processes implicated in ADHD. Prevailing theoretical models of ADHD postulate that executive dysfunction and a strong preference for immediate over delayed reward (e.g., delay discounting) underlie difficulties with behavioral control characteristic of ADHD (Barkley, 1997; Sagvolden, Johansen, Aase, \& Russell, 2005; Sonuga-Barke, 2002). Based on previous research demonstrating that working memory is related to VDAC among healthy adults (Anderson et al., 2011b; Anderson \& Yantis, 2012) and evidence of working memory deficits in ADHD (see meta-analysis by Martinussen, Hayden, Hogg-Johnson, \& Tannock, 2005), working memory may contribute to VDAC among children with ADHD. In addition, delay discounting, a motivational process that is implicated in theories of ADHD (Sagvolden et al., 2005; Sonuga-Barke, 2003; Sonuga-Barke, Bitsakou, \& Thompson, 2010) and supported by the empirical literature (see meta-analysis by Patros et al., 2016) may be 
related to VDAC. One possibility is that the heightened sensitivity to immediate reward (i.e., greater delay discounting) often observed in children with ADHD places greater demands on executive control to maintain attention to the primary task in the presence of valuable or rewarding distractors. Thus, it is reasonable to suspect separate and interactive contributions of working memory and delay discounting to increased distractibility to rewarding stimuli in ADHD as reflected in VDAC.

The current study is the first to our knowledge to directly examine the extent to which children with ADHD are more distracted by rewarding stimuli with a well-validated experimental paradigm. Specifically, we compared the performance of children with ADHD and typically developing controls on the VDAC paradigm, which measures the extent to which an irrelevant non-salient distractor, previously associated with reward, captures attention (Anderson et al., 2011a, 2011b; Anderson \& Yantis, 2012; Sali, Anderson, \& Yantis, 2014). Given evidence that children with ADHD are more sensitive to reward outcomes than are their typically-developing peers, one possible outcome is that participants with ADHD will show greater VDAC than will control participants. However, just as children with ADHD discount the future value of reward relative to their typicallydeveloping peers, it is also possible that ADHD will be associated with a reduction in the magnitude of capture since the distractor stimuli are goal-irrelevant and no longer predict rewards. Finally, previous studies have shown that children with and without ADHD demonstrate similar degrees of saliency-driven attentional capture (Van der Stigchel et al., 2007) and thus we may find no difference among diagnostic groups in VDAC. Lastly, given the possibility that children with and without ADHD may demonstrate extinction of reward learning to different extents, all analyses are presented both collapsed across the entirety of the experiment and broken down by experimental half. The results of the current study will therefore shed light on how reward learning influences future settings of attentional priority in ADHD. Further, we examined associations with ADHD symptom severity and the mechanisms underlying distractibility to reward in ADHD by evaluating whether VDAC correlates with working memory and delay discounting. We hypothesized that greater VDAC would correlate with weaker working memory and greater delay discounting. Importantly, if there is heterogeneity in the magnitude of VDAC among participants with ADHD, it is possible that these correlational analyses will detect relationships among VDAC and working memory or delay discounting even if there is no significant difference based on diagnosis at the group level.

\section{Method}

\section{Participants}

The VDAC paradigm was administered to 56 children (ages 8-12 years), including 30 participants diagnosed with ADHD and 26 typically developing (TD) controls, who participated in a larger study comparing children with ADHD to TD children on various neuropsychological assessments and neuroimaging measures. Participants were primarily recruited through local schools, with additional resources including community-wide advertisement, volunteer organizations, medical institutions, and word of mouth. This study was approved by the Johns Hopkins University School of Medicine Institutional Review 
Board and all data was obtained in compliance with their regulations. After complete description of the study to the participants, written informed consent was obtained from a parent/guardian and assent was obtained from the child.

To determine study eligibility, an initial screening was conducted through a telephone interview with a parent. Children with a history of intellectual disability, learning disability, seizures, traumatic brain injury or other neurological illnesses were excluded from participation. Intellectual ability was assessed using the Wechsler Intelligence Scale for Children, Fourth and Fifth Editions (Wechsler, 2003, 2014) and participants with full-scale intelligence quotient (FSIQ) scores below 80 were excluded. In addition to inquiring about a history of a learning disability, children were also administered the Word Reading subtest from the Wechsler Individual Achievement Test, Second Edition (Wechsler, 2002) to further screen for a reading disorder and were excluded for Word Reading scores below a standard score of 85 .

Diagnostic status was established through administration of either the Diagnostic Interview for Children and Adolescents ( $n=26$ ), Fourth Edition (DICA-IV; Reich, Welner, \& Herjanic, 1997) which follows DSM-IV criteria for all disorders (American Psychiatric Association, 1994) or the Kiddie Schedule for Affective Disorders and Schizophrenia for School Aged Children Present Lifetime version ( $n=30$ ) (Kaufman et al., 2013) which follows DSM-5 criteria for all disorders (American Psychiatric Association, 2013). Children meeting criteria for diagnosis of conduct, mood, generalized anxiety, separation anxiety or obsessivecompulsive disorders based on the diagnostic interview were excluded. A comorbid diagnosis of oppositional defiant disorder (ODD) was permitted given the high base rate comorbidity between ADHD and ODD. Parents and teachers (when available) also completed the Conners' Parent and Teacher Rating Scales-Revised Long Version $(n=2)$ (CPRS and CTRS; Conners, 1997) or the Conners-3 ( $n=54$ ) (Conners, 2008) and the ADHD Rating Scale-IV, home and school versions (DuPaul, Power, Anastopoulos, \& Reid, 1998). Although teacher report was not always available, we obtained information from the parent about the child's symptoms and functioning at school during the diagnostic interview with parents. A child would only meet diagnostic criteria for ADHD if the primary caregiver reported ADHD symptoms and associated impairment at home and at school or in other settings. This information was then reviewed and the diagnosis was confirmed by a child neurologist or psychologist.

An ADHD diagnosis was established based on the following criteria: (1) T-score of 65 or higher on the ADHD Inattentive or Hyperactive/Impulsive scales on the CPRS or CTRS, when available, or a raw score of 2 or 3 on at least 6/9 items on the Inattentive or Hyperactivity/Impulsivity scales of the ADHD-RS and (2) an ADHD diagnosis on the DICA-IV or KSADS-PL. This information was then reviewed and the diagnosis was confirmed by a child neurologist or psychologist based on DSM-5 criteria. Children taking psychotropic medications other than stimulants were excluded from participation and all children taking stimulants were asked to withhold their medication the day of and day prior to the laboratory visit, as in prior work including children on long-acting stimulants. 
Inclusion in the control group required scores below clinical cutoffs on the parent and teacher (when available) rating scales (CPRS, CTRS, and ADHD-RS). Control participants could not meet diagnostic criteria for any psychiatric disorder based on DICA-IV or KSADS-PL nor could they have history of neurological disorder, learning disability, or be taking any psychotropic medication. They were also required to have an FSIQ of at least 80 on the WISC and a score of at least 85 on the WIAT-II word reading subtest. Finally, children with a history of color blindness were excluded from participation and the Ishihara test of color blindness was administered as an additional screening measure on the first visit to the laboratory.

Participation in the current study was part of a larger multi-session battery involving neuropsychological tasks and neuroimaging administered over the course of two to three laboratory visits lasting from approximately 8:30 AM to 3:30 PM. The VDAC paradigm was administered on the second testing day for the majority of participants and occasionally on the third testing day for children who participated in multiple studies. Participants were offered frequent breaks throughout the day to prevent fatigue and maintain motivation. The order of tasks administered varied across participants alternating between computer-based tasks and more active tasks, such as motor assessments. The VDAC paradigm included two training sessions followed by a testing session administered over the course of the morning (between 8:30 AM 12:00 PM) with various tasks administered in between the training and test sessions. ${ }^{1}$ Research assistants administering the VDAC had either a Bachelors or Masters degree and were well-trained on testing pediatric populations in general and the VDAC task in particular. The experimenter carefully reviewed a set of standardized instructions with participants and would only proceed with the task once the participants could accurately explain the task. Participants were compensated for their time ( $\$ 50$ per day) in addition to the money earned during the VDAC task, which was added to their check upon completion of the study.

\section{Value-Driven Attentional Capture Task}

Training Phase-Participants first completed a visual search task (Psychophysics toolbox, Matlab) in which the target stimulus's color predicted the magnitude of trial-by-trial monetary rewards. On each trial, participants viewed a search array that consisted of six colored circles $\left(4.76^{\circ} \times 4.76^{\circ}\right)$ arranged along the outside of an imaginary circle with a radius of approximately $8^{\circ}$ around a central fixation cross $\left(1.59^{\circ}\right.$; see Figure 1a) on a computer monitor positioned approximately $70 \mathrm{~cm}$ from the participant. The target in each array was defined as the circle that was either red or green, and participants were informed that there would never be both a red and a green circle present in the same array. The colors of the nontarget circles were randomly selected on each trial from blue, yellow, white, orange, cyan, and purple. The target in each array contained an arrow line segment that pointed to either the left or to the right. For the nontarget stimuli, the arrows were bidirectional. Participants were instructed to press the $\mathrm{Z}$ key (labeled with a left-pointing arrow) of a standard computer keyboard if the enclosed arrow pointed to the left or the $\mathrm{M}$

\footnotetext{
${ }^{1}$ One participant in the control group completed the second training session and the testing session on a separate day from the first training session due to a power outage. This participant's overall value-driven attentional capture score was less than $0.5 \mathrm{SD}$ below the mean of all control participants and was therefore not an outlier in the final sample.
} 
key (labeled with a right-pointing arrow) if the arrow pointed to the right. As in earlier studies (Anderson et al., 2011a; Sali et al., 2014), participants received monetary rewards for correctly identifying the orientation of the arrow in the target stimulus. All earnings were added to the value of the check they received as compensation. Critically, the target color (red or green) predicted whether the participant would receive a high (8 cents) or low (2 cents) magnitude reward on $80 \%$ versus $20 \%$ of the trials, respectively. For 24 participants, a red target was associated with the potential for a high magnitude reward on $80 \%$ of trials and a low magnitude reward on the remaining $20 \%$ of trials, while a green target was associated with a low magnitude reward on $80 \%$ of trials and a high magnitude reward on the remaining $20 \%$ of trials. The remainder of the participants received the opposite target-color reward magnitude probability contingencies. Although the test phase ultimately only included the high-magnitude reward-associated stimulus, it is important that both high and low magnitude reward stimuli were included in the traning phase since previous studies have shown that VDAC occurs only when stimulus features predict trial-by-trial reward outcomes (Sali et al., 2014). If only the high-value target was included in the training phase, stimulus color would not uniquely predict reward outcomes above and beyond any of the other stimulus features and thus participants would not learn the stimulus-reward association.

Trial structure was as follows: fixation cross (variable 400-600 ms), search array (1500 ms), blank screen (1000 ms), feedback (money earned per trial and running total; $1500 \mathrm{~ms}$ ), and blank screen $(1000 \mathrm{~ms})$. Incorrect responses or responses $>1500 \mathrm{~ms}$ produced no feedback or monetary gain. In order to increase the salience of the reward magnitude difference, low and high rewards were always accompanied by a single or double presentation of a cash register "cha-ching" sound, respectively. For trials in which participants failed to respond, they heard a $1000 \mathrm{hz}$ tone for $250 \mathrm{~ms}$. To maximize participants' potential to learn the reward associations between target color and monetary reward, participants completed two separate training sessions of 120 trials each ( $\sim 15$ minutes) within the same day of testing with one self-paced break halfway through each session and a longer break in-between sessions. Thus, the current paradigm was closely matched to previous studies of VDAC in healthy adults (e.g., Anderson et al., 2011b) with the exception of the following modifications to make the task more appropriate for children while still maintaining the main task components: (1) longer stimulus presentation (as in, e.g., Anderson, Faulkner, Rilee, Yantis, \& Marvel, 2013), (2) additional cash register sound during reward feedback presentation, (3) the addition of a second training session to maximize the potential of reward learning (as in, e.g., Laurent, Hall, Anderson, \& Yantis, 2015), and (4) participants were instructed to respond based on the direction of the enclosed arrow rather than the orientation of a line segment.

Test Phase-Immediately following the second training session, all participants completed an additional visual search task that was designed to test whether the training phase association between color and reward differentially modulated attentional priority across the two participant groups. Critically, participants now searched for a shape singleton in each array, which was either a diamond among circles or a circle among diamonds, and again reported the orientation of the enclosed arrow line segment (see Figure 1b). As before, the nontarget stimuli encompassed bidirectional arrows so that they were not preferentially 
associated with either direction. Importantly, on half of the trials, one of the nontarget items appeared in the previously high value reward-associated color (distractor present trials), and on the remainder of trials, the high-value reward-associated color was absent from the array (distractor absent trials). In order to maximize the possibility of detecting value-driven attentional capture while limiting the duration of the test phase to prevent fatigue, we restricted this manipulation to the high value color from training and consequently, the low value color never appeared during the test phase. The test phase target stimulus never appeared in a previously rewarded color so that current search goals (e.g. find the unique shape) were never compatible with attentional selection of the previously rewarded item. The test phase paradigm therefore provided a means to test the degree to which rewardassociated stimuli involuntarily captured attention (as reflected in a slowing of response time on distractor present trials) when divorced from behavioral goals.

In order to ensure that participants remained highly motivated to respond as quickly and accurately as possible during the test phase, we again employed a reward manipulation (e.g., Anderson, Laurent, \& Yantis, 2013). The new reward manipulation was orthogonal to the associations set up during the training phase such that target shape, rather than target color, predicted the magnitude of subsequent reward outcomes and used reduced reward magnitudes relative to training so as to minimize the possibility of overriding the training phase learning. Specifically, for approximately half of the participants in each diagnostic group, a diamond target was associated with a high magnitude reward of 4 cents and a circle target was associated with a low magnitude reward of 1 cent on $80 \%$ of all trials. These reward magnitudes were reversed on the remaining trials. All other participants received the opposite shape-reward magnitude contingencies.

The test phase consisted of a single session of 120 trials with similar structure to the training sessions (see Figure 1b) and lasted for approximately 15 minutes. Participants again received a single self-paced break in the middle of the test phase task. The test phase was thus similar to previous studies of healthy adults, but included modifications that were related to those of the training phase, such as an increase of trial length and the use of arrow stimuli to simplify the stimulus-response mapping. The increase in response times for distractor present trials relative to distractor absent trials, a difference that can only be attributed to learned reward associations, served as the primary metric of interest.

\section{Spatial Span Task}

Visual-spatial working memory was assessed via a computerized adaptation (Shiels et al., 2008) of the spatial span subtest from the WISC-IV or WISC-V Integrated that incorporated features of the spatial span task from the CANTAB (Luciana, 2003). In this task, an array of ten white squares on a black background is presented on the computer screen. On each trial, a yellow smiley face appears in two to eight of the squares at a rate of one square per second. Children were instructed to use a computer mouse to click on the squares in the same order in which the smiley face appeared (forward span) or in the reverse order (backward span), the latter requiring manipulation of visual-spatial information. Forward span was always administered before backward span. For each direction (forward and backward), there were two trials at each level of difficulty, beginning with two-location 
sequences and advancing to a maximum of eight-location sequences. The task terminated when both trials within a difficulty level were incorrect. Task duration varied across subjects depending on performance but it typically ranged from 5-15 minutes. The primary measure of interest from this task is the total number of trials completed correctly for the backward span with a higher score indicative of better working memory.

\section{Monetary Delay Discounting Task}

Participants completed a computer-based delay discounting task (Rosch \& Mostofsky, 2016; Wilson, Mitchell, Musser, Schmitt, \& Nigg, 2011) involving 91 choices between a varying amount of money now ( $\$ 0-\$ 10.50$ in $\$ 0.50$ increments) or $\$ 10.00$ after a varying delay (1, 7,30 , or 90 days) in which some of the choices were real (i.e., participants receive the amount of money at the chosen delay for two randomly-selected choices in the form of gift cards or prizes). As in previous research (Rosch \& Mostofsky, 2016), an indifference point was identified for each delay to determine the area under the curve (Myerson, Green, \& Warusawitharana, 2001), with smaller values indicating greater delay discounting thought to reflect a stronger preference for immediate reward.

\section{Data Reduction and Analysis}

A total of 8 participants (6 ADHD, 2 control) were excluded from all analyses for having overall behavioral accuracies in the test phase of the VDAC task falling below $70 \%$. To ensure that we had sufficient power to detect a difference in the magnitude of VDAC between children with and without ADHD, we conducted a power analysis. Although our study is the first to test VDAC in children with ADHD, two recent studies have compared the magnitude of VDAC in healthy controls to inviduals with depression (Anderson, Leal, Hall, Yassa, \& Yantis, 2014) and drug addiction (Anderson, Faulkner, et al., 2013). When collapsing data across both studies and focusing on the comparison of high value distractor present trials versus distractor absent (the comparison most relevant to the current study), we found an effect size, as indexed by partial eta-squared, of $\eta_{\mathrm{p}}=.161$. Applying this effect size to a power analysis in $\mathrm{G}^{*}$ Power (Faul, Erdfelder, Lang, \& Buchner, 2007) indicated that we would need 46 participants to reach $80 \%$ power. Thus, our final sample of 48 participants is sufficient to detect a group difference in VDAC scores between children with and without ADHD.

For each participant, all RTs greater than 2.5 standard deviations above or below the mean of each condition were excluded from the analysis. Together, this procedure resulted in a loss of less than $2 \%$ of all trials across all participants. In order to test whether previous reward learning differentially modulated the setting of attentional priority in children with and without ADHD, we employed three main analyses. First, using a 2 diagnostic group (ADHD vs. control) $\times 2$ target value (low value vs. high value) $\times 2$ training session factorial analysis of variance (ANOVA), we tested whether behavioral accuracies varied across diagnostic groups during training. This analysis allowed us to rule out the possibility that differences in training performance, and thus the extent of reward feedback received, could account for test phase differences in attentional capture. Next, we tested for differences in behavioral accuracy in the test phase with a 2 distractor presence (present vs. absent) $\times 2$ diagnostic group (ADHD vs. control) ANOVA. Finally, as our primary measure of interest, we 
computed an index of attentional capture for each participant by subtracting mean response times (RTs) for distractor absent trials from distractor present trials in the test phase, referred to below as a VDAC score. We then subjected these scores to an additional 2 diagnostic group $\times 2$ experimental half (first vs. second) ANOVA to determine whether the degree to which the reward associated distractor captured attention varied across groups and time. All post-hoc $t$-test comparisons of the training and test phase VDAC data were corrected for multiple comparisons by controlling the false discovery rate according to a linear step-up procedure as implemented in Matlab R2014B (Benjamini \& Hochberg, 1995). All reported $t$-test $\mathrm{p}$-values have been corrected accordingly and significance was determined at $\mathrm{q}=.05$.

Lastly, we ran a series of exploratory correlational analyses to quantify the relationship between VDAC and (1) dimensional measures of ADHD symptom severity (parent-reported ADHD rating scale raw scores) among children with ADHD and (2) measures of working memory and delay discounting within each diagnostic group for a subset of participants who completed these additional tasks. Given that correlations are more susceptible to outliers than the main analysis described above, we adopted an outlier removal procedure for the correlational analyses linking the magnitude of VDAC to working memory, delay discounting and symptom severity. For each of the task measures, as well as for the VDAC task, we excluded participants who fell more than 2.5 standard deviations away from the mean of their respective diagnostic group. Due to the small sample size and to maximize our power, we conducted this outlier removal procedure on a test-by-test basis. Applying this criterion resulted in the exclusion of two participants for the working memory correlation, one participant for the delay discounting correlation, and one participant for the symptom severity correlation. Given the small sample size for each task correlational test (ranging from $n=20$ to $n=23$ per diagnostic group), we discuss the magnitude of the relationship such that small, medium, and large effects are represented by $r=.10, .30$, and .50 , respectively. $T$-tests evaluating differences in WM and delay discounting scores across diagnostic groups were not included in the FDR correction procedure described above because they were planned comparisons of independent data. Lastly, we report partial etasquared and Cohen's $d$ as measures of effect size for all ANOVA and $t$-test results, respectively. For Cohen's $d$ we consider $d=0.2$ to be a "small" effect, $d=0.5$ to be a "medium" effect, and $d=0.8$ as a "large" effect. For partial eta-squarred, we consider $\eta_{\mathrm{p}}=$. 01 to be a "small" effect, $\eta_{\mathrm{p}}=.09$ to be a "medium" effect, and $\eta_{\mathrm{p}}=.25$ to be a "large" effect.

\section{Results}

\section{Sample Characteristics}

Demographic information for the sample included in the primary VDAC analyses is provided in Table 1, along with inferential statistics regarding diagnostic group differences. The sample was drawn from largely middle class socioeconomic status (Hollingshead, 1975) and was 63\% caucasian. Diagnostic groups did not significantly differ in several important demographics including age, sex, ethnicity, socioeconomic status and intellectual reasoning ability. 


\section{Training Phase Performance}

We first examined the behavioral accuracies from the initial training phase sessions in which target stimulus color predicted trial-by-trial reward magnitudes. Since participants only received monetary rewards for correct responses, significant differences in training phase performance across the two diagnostic groups could account for differences in subsequent test phase VDAC. Importantly, there was no significant main effect of diagnostic group, $F(1,46)=1.25, p=.269, \eta_{\mathrm{p}}=.026$, ruling out the possibility that differences in subsequent test phase attentional capture could be attributed to differences in training phase performance, and behavioral accuracies were overall very good (approximating 90\%) for both diagnostic groups (see Figure 2a). The main effect of session, $F(1,46)=3.59, p=.065$, $\eta_{\mathrm{p}}=.072$, the main effect of target value, $F(1,46)=3.20, p=.080, \eta_{\mathrm{p}}=.065$, and the threeway interaction of Diagnostic Group $\times$ Session $\times$ Target Value, $F(1,46)=3.29, p=.076, \eta_{\mathrm{p}}$ $=.067$, approached statistical significance. Although the three-way interaction was not significant, we were particularly interested in the performance of children with ADHD and controls on high value trials during session two to determine whether diagnostic groups had similar exposure to the high value reward outcome prior to beginning the test phase. We found that behavioral accuracy did not differ between ADHD and control participants for high value trials during training session $2, t(46)=0.33, p=.795, d=0.10$, suggesting similar exposure to reward outcomes just prior to the test phase. All other main effects and interactions failed to reach statistical significance, $F_{\mathrm{s}}<0.898, p \mathrm{~s}>.348$ (see Table 2).

\section{Test-Phase Performance}

We next examined test phase behavioral accuracies. Overall, test phase accuracies were greater for control participants than for ADHD participants, $F(1,46)=5.33, p=.025, \eta_{\mathrm{p}}=$. 104. However, there was no evidence of a significant main effect of distractor, $F(1,46)=$ $2.40, p=.128, \eta_{\mathrm{p}}=.050$, or a distractor presence by diagnostic group interaction, $F(1,46)=$ $0.01, p=.944, \eta_{\mathrm{p}}<.001$, suggesting that the RT results were not due to a speed accuracy tradeoff (see Figure 2b).

Next, we tested whether participants were slowed by the presence of a previously rewarded distractor in the search array and whether the extent of slowing differed for participants with and without ADHD over the course of the test phase. For these analyses, we calculated a difference score reflecting the degree to which the previously rewarded distractor slowed participants (see Data Reduction and Analysis). The results of the 2 diagnostic group $\times 2$ experimental half (first vs. second) ANOVA indicated a main effect of diagnostic group, $F(1,46)=14.97, p<.001, \eta_{\mathrm{p}}=.246$, such that control participants, $t(23)=5.63, p<.001, d$ $=1.15$, but not those with ADHD, $t(23)=0.26, p=.795, d=.05$, demonstrated significant value driven attentional capture when collapsing across the entire test phase (Figure 3).

Due to the possibility of differences in the rate of extinction of learning across the two diagnostic groups, we probed changes in VDAC scores over the course of the test phase for both groups. A main effect of experimental half indicated that, regardless of diagnostic group, participants were less distracted by the reward-associated distractor in the second half of the test phase than in the first half, $F(1,46)=5.76, p=.021, \eta_{\mathrm{p}}=.111$, providing evidence in favor of extinction of learning. The interaction of diagnostic group by experimental half 
failed to reach statistical significance, $F(1,46)=0.62, p=.436, \eta_{\mathrm{p}}=.013$, suggesting that the rate of extinction did not reliably vary based on diagnosis. However, further probing of changes in VDAC over the test phases revealed the following pattern. During the first half of test phase trials when the strength of learning was presumably the strongest prior to any extinction, both control, $t(23)=5.15, p<.001, d=1.05$, and ADHD, $t(23)=2.32, p=.047$, $d=0.47$, participants were significantly slower for trials in which the previously rewarded distractor was present in the search array than when it was absent, suggesting that participants in both groups formed an initial reward association. Moreover, VDAC scores were greater for control than for ADHD participants in the first half of trials alone, $t(46)=$ 2.22, $p=.047, d=.64$. In contrast, during the second half of the test phase, only control participants showed significant VDAC, $t(23)=3.68, p=.004, d=0.75$, whereas children with ADHD did not, $t(23)=-1.09, p=.368, d=0.22$. VDAC scores were again greater for control than for ADHD participants, $t(46)=2.79, p=.017, d=0.81$. This pattern of results suggest that although children with and without ADHD both showed evidence of value driven attentional capture early in the test phase, this capture was stronger in control participants than in those with ADHD throughout the test phase.

\section{Correlations with ADHD Symptoms}

Among children with ADHD, we found a non-significant correlation with parent-reported total ADHD symptoms raw score $(r(21)=-.21, p=.336)$, although it was in the expected direction (i.e., greater VDAC was associated with fewer ADHD symptoms). When examining VDAC in relation to each individual symptom domain, there was a marginally significant correlation with hyperactive/impulsive total score $(r(21)=-.39, \mathrm{p}=.063$; medium effect $)$, but not with inattentive symptoms $(r(21)=.197, \mathrm{p}=.367)$.

\section{Correlations with Working Memory and Delay Discounting Tasks}

Lastly, we examined bivariate correlations between VDAC and individual differences in working memory and delay discounting separately among children with ADHD and controls (see Figure 4). Among controls, there was no evidence of a relationship between VDAC and either working memory, $r(18)=-.11, p=.653$, or delay discounting, $r(21)=-.01, p=.967$. Within the ADHD group, greater VDAC scores correlated with backward span, $r(19)=-.44$ (medium effect), $p=.045$ (see Figure 4a), but not with monetary delay discounting (AUC), $r(19)=-.04, p=.855$ (see Figure $4 \mathrm{~b}$ ). This pattern of correlation suggests that participants with ADHD with the weakest spatial working memory capacity were slowed the most by the presence of a previously rewarded distractor. Furthermore, immediate reward preference was unrelated to the degree to which participants with ADHD were slowed by the presence of a previously rewarded distractor. Of note, children with ADHD and their TD peers did not significantly differ in their performance on the backward span, $t(39) 1.63, p=.111, d=.51$, or delay discounting tasks, $t(42)=-.49, p=.624, d=.15$, with medium and small effect sizes observed, respectively.

\section{Discussion}

In the current study, we found that children with ADHD were significantly less slowed by the presence of a previously reward-associated distractor in a visual search task than were 
their typically-developing peers. Critically, as in earlier studies (Anderson et al., 2011b), these reward-associated distractors were always goal-irrelevant and did not differ from reward-neutral distractor items in low-level physical characteristics. Thus, any slowing due to the presence of the previously rewarded distractor can be attributed to the learned reward associations rather than to goal-oriented or stimulus-driven attentional orienting processes (Sali et al., 2014). However, children with ADHD were still significantly slowed by the presence of the distractor in the first half of the test phase when reward associations were likely the strongest. Participants in both diagnostic groups therefore formed reward associations that modulated settings of attentional priority, making our results consistent with earlier accounts applying the VDAC paradigm to healthy adolescents (Roper, Vecera, \& Vaidya, 2014).

Our finding of less VDAC in children with ADHD in comparison to control participants failed to support our predictions that reward learning may contribute to symptoms of inattention in ADHD by boosting the attentional priority of goal-irrelevant, but high valueassociated, stimuli. Theoretical models of ADHD emphasize altered reinforcement sensitivity (see review by Luman et al., 2010), although the nature of the reinforcement deficit remains unclear due to inconsistencies in behavioral and neuroimaging findings. In general, there is evidence that children with ADHD display a stronger preference for immediate over delayed rewards than do typically developing children (Patros et al., 2016) and improved cognitive performance in response to reward (e.g., Fosco, Hawk, Rosch, \& Bubnik, 2015; Rosch et al., 2016), as well as atypical activation of reward circuitry (see meta-analysis by Plichta \& Scheres, 2014). In contrast to our predictions, we found that children with ADHD were less distracted by stimuli previously associated with reward. One possible explanation for reduced VDAC is that children with ADHD did not learn the reward associations to the same degree as did control participants despite similar training exposure. However, the presence of VDAC in participants with ADHD at the beginning of the test phase suggests that our ADHD group did learn the reward association. Alternatively, it is possible that children with ADHD were less distracted by the reward-associated stimulus because it did not predict test phase reward outcomes; instead, new stimulus-reward contingencies were introduced to maintain task engagement. Children with ADHD may have focused on earning rewards in the test phase and failed to maintain the value of the previously reward-associated stimulus, resulting in less VDAC than their typically developing peers. The reward associations acquired during the training phase may also extinguish more rapidly among children with ADHD such that once a stimulus is no longer rewarded, it loses its value more quickly. Although the diagnostic group by test phase half interaction was not significant in the current study, it will be important to replicate and extend these findings in a larger sample. Furthermore, delay discounting was not correlated with VDAC among children with ADHD, suggesting that these behavioral indicators may reflect different components of reward sensitivity implicated in ADHD.

As in earlier studies of healthy adults (Anderson et al., 2011b; Anderson \& Yantis, 2012), we also observed a significant relationship between the magnitude of VDAC and spatial working memory capacity in children with ADHD. Poorer working memory was associated with more pronounced VDAC among children with ADHD. Interestingly, we did not observe this pattern for control participants, who showed greater VDAC as a group and 
generally show greater working memory capacity (see Anderson, Faulkner, et al., 2013 for a similar finding in drug addiction). This pattern of findings may reflect the heterogeneity of cognitive and motivational deficits in children with ADHD, such that while we may not see greater VDAC in the ADHD group as a whole compared to controls, those children with ADHD with greater WM impairments show greater VDAC. There are few studies examining working memory in relation to VDAC, and the present study is the first to do so among children and among individuals with ADHD. Individuals with poorer working memory may have more difficulty controlling their attention in the face of distraction and are therefore more distracted by stimuli previously associated with reward (Anderson et al., 2011b; Fukuda \& Vogel, 2011). In particular, spatial working memory capacity has been linked to the ability to resist attentional capture, and thus, our correlational analysis focused on the test phase in which attentional capture is measured. At this time, it is unclear whether other executive functions, such as response inhibition and attention regulation, relate to VDAC. This will be an important question for future research. It is also important to acknowledge research suggesting that performance on memory span tasks may reflect short-term memory rather than working memory (Engle, Tuholski, Laughlin, \& Conway, 1999), although it is unclear whether this is true for visual-spatial tasks as this literature is based on verbal span tasks. Thus, it will be important for future research to examine more complex span tasks and other validated measures of visual-spatial working memory to expand upon these preliminary findings.

Unlike most previous studies, we observed significant extinction of learning for both control and ADHD participants over the course of the test phase. VDAC in adults has been shown to persist throughout experimental sessions longer than that used in the current study (Anderson et al., 2011a, 2011b; Anderson \& Yantis, 2012), although other studies have also shown extinction of VDAC within the test phase among healthy adults (Roper et al., 2014). Furthermore, VDAC has even been observed when participants completed a test phase similar to that used in the current experiment 6 months after the initial reward learning (Anderson \& Yantis, 2013). Thus, in adults, value-based attentional priority persists long after these associations are no longer goal-relevant. Conversely, our study suggests that, regardless of diagnostic group, the modulatory role of reward associations in attentional control in children is potentially more vulnerable to extinction over time. However, it is also important to recognize that the test phase in our paradigm differed from that of most previous studies in that participants were rewarded for performance (see Anderson, Laurent, et al., 2013 for a similar reward at test paradigm). The introduction of new stimulus-reward associations may increase the rate of extinction for the stimulus-reward associations learned during the training phase. However, this paradigm change was important in order to maintain task engagement due to pilot data suggesting a large decline in performance once rewards could no longer be earned during the test phase relative to the training phase, especially among children with ADHD who may be particularly sensitive to the removal of rewards (e.g., Shiels et al., 2008).

The robust difference in the degree of VDAC between children with and without ADHD in the current study provides further evidence that VDAC operates through mechanisms that are distinct from those that are implicated in goal-oriented and stimulus-driven attentional control. Specifically, well-validated measures of visual search from basic science thought to 
relate to inattention symptoms have largely failed to detect any diagnostic group differences. For example, while children with ADHD are slower to identify a target stimulus among distractors, the relative slowing associated with the introduction of additional distractors is equivalent to that in typically developing children (Hazell et al., 1999; Mason, Humphreys, \& Kent, 2004; Mason, Humphreys, \& Kent, 2003). Moreover, children with ADHD are equivalently slowed by the presence of a physically salient distractor in tests of covert and overt attentional orienting (Van der Stigchel et al., 2007). Thus, goal-oriented and stimulusdriven attentional orienting mechanisms appear to be similar in children with and without ADHD. Conversely, a growing body of literature has illustrated the link between VDAC and reinforcement learning. Specifically, VDAC only occurs in situations in which stimulus features generate reward prediction errors (Sali et al., 2014), has been linked to activation of the midbrain and striatum rather than frontoparietal brain regions (Anderson, 2017; Anderson, Kuwabara, et al., 2016; Anderson et al., 2017; Anderson, Laurent, \& Yantis, 2014), and occurs even for stimuli that are never goal-relevant (Le Pelley, Pearson, Griffiths, $\&$ Beesley, 2015). Taken together, the observed diagnostic group difference in VDAC provides additional evidence that reward learning shapes settings of attentional priority in a way that is distinct from behavioral goals and stimulus salience and may be differentially affected in ADHD.

The current study was designed to provide an initial test of the role reward learning plays in the rapid instantiation of attentional priority in children with ADHD, and thus, future research is needed to expand on our findings and address several limitations. First, the small sample recruited in the current study is not well-suited for testing whether the magnitude of VDAC varies across ADHD subtypes and genders. In the current sample, roughly $30 \%$ of participnats had ADHD-Inattentive, whereas 70\% had ADHD-combined. A replication of our findings in a larger sample would therefore extend our understanding of the group difference observed here in VDAC magnitude between TD children and those with ADHD. Furthermore, the exclusion criteria of the current study were set based on a larger battery of neuropsychological and neuroimaging tasks for which the current data are a small subset. While we set our exclusion criteria based on low FSIQ, this likely eliminated individuals with poorer processing speed and working memory performance often observed in children with ADHD, which may have weakened the relationship between working memory and VDAC. Future research should consider excluding participants based on the general ability index (GAI), an estimate of intellectual reasoning ability that does not factor in performance on the processing speed and working memory tests, rather than FSIQ. Lastly, it will be important for future research to consider other cognitive deficits that might be related to variability in VDAC among children with and without ADHD in addition to working memory and delay discounting. By studying the relationship between VDAC and other measures of the component processes of executive functioning, future research will clarify the reasons why some individuals experience greater VDAC than do others.

Children with ADHD included in the current study were diagnosed with limited comorbid conditions (i.e., ODD). Therefore, findings might not generalize to a more severe/clinical ADHD population. Inclusion of additional, lower base rate comorbidities (e.g., major depressive disorder, anxiety disorders) in future studies will serve to increase generalizability of findings to clinical populations of children with ADHD. 
In the current study, we examined the extent to which reward-associated stimuli involuntarily capture the focus of attention in children with ADHD in comparison to their typically developing peers. Using the VDAC paradigm, we found that children with ADHD demonstrated significantly less attentional capture by stimuli previously associated with reward than did control participants. Our results contribute a novel behavioral finding to the empirical literature characterizing atypical response to reward in ADHD by demonstrating that ADHD may be associated with a decrease in the degree to which goal-irrelevant reward associations influence the setting of attentional priority. This finding might suggest that heightened distractibility outside of the laboratory among individuals with ADHD may not be due to the presence of stimuli previously associated with reward when a reward is offered for the primary task, as in the current study. Clinically, this may mean that rewarding a target behavior (e.g.. performance of a particular task), as is often done in behavioral contingency management treatment, may effectively reduce distractibility among children with ADHD. It may also be that children with ADHD fail to maintain stimulus-reward associations once new contingencies are in place, focusing instead on the currently available rewards in their environment. This also has important clinical implications as a possible explanation for the finding that problematic behavior often re-emerges upon discountinuation of the behavioral treatment involving reward and punishment contingencies. Thus, there are several important questions to be addressed in future research aimed at elucidating the mechanisms of diminished value-driven attentional capture among children with ADHD and its relevance to real-world distraction in ADHD.

\section{Acknowledgments}

Funding: This work was supported in part by grants from the National Institute of Mental Health (RO1 MH078160; RO1 MH085328, K23 MH101322), the Johns Hopkins University School of Medicine Institute for Clinical and Translational Research National Institutes of Health/National Center for Research Resources Clinical and Translational Science Award program UL1 TR 000424-06, and the Kennedy Krieger Institute/Johns Hopkins University Intellectual and Developmental Disabilities Research Center (IDDRC; U54 HD079123).

\section{References}

American Psychiatric AssociationDiagnostic and statistical manual of mental disorders 4. Washington, DC: American Psychiatric Association; 1994

American Psychiatric AssociationDiagnostic and statistical manual of mental disorders 5. Arlington, VA: American Psychiatric Association; 2013

Anderson BA. Reward processing in the value-driven attention network: reward signals tracking cue identity and location. Soc Cogn Affect Neurosci. 2017; 12(3):461-467. DOI: 10.1093/scan/nsw141 [PubMed: 27677944]

Anderson BA, Faulkner ML, Rilee JJ, Yantis S, Marvel CL. Attentional bias for nondrug reward is magnified in addiction. Experimental and Clinical Psychopharmacology. 2013; 21(6):499-506. DOI: 10.1037/a0034575 [PubMed: 24128148]

Anderson BA, Kronemer SI, Rilee JJ, Sacktor N, Marvel CL. Reward, attention, and HIV-related risk in HIV+ individuals. Neurobiology of Disease. 2016; 92(Pt B):157-165. DOI: 10.1016/j.nbd. 2015.10.018 [PubMed: 26484383]

Anderson BA, Kuwabara H, Wong DF, Gean EG, Rahmim A, Brasic JR, Yantis S. The Role of Dopamine in Value-Based Attentional Orienting. Curr Biol. 2016; 26(4):550-555. DOI: 10.1016/ j.cub.2015.12.062 [PubMed: 26877079]

Anderson BA, Kuwabara H, Wong DF, Roberts J, Rahmim A, Brasic JR, Courtney SM. Linking dopaminergic reward signals to the development of attentional bias: A positron emission 
tomographic study. Neuroimage. 2017; 157:27-33. DOI: 10.1016/j.neuroimage.2017.05.062 [PubMed: 28572059]

Anderson BA, Laurent PA, Yantis S. Learned value magnifies salience-based attentional capture. PLoS One. 2011a; 6(11):e27926.doi: 10.1371/journal.pone.0027926 [PubMed: 22132170]

Anderson BA, Laurent PA, Yantis S. Value-driven attentional capture. Proc Natl Acad Sci U S A. 2011b; 108(25):10367-10371. DOI: 10.1073/pnas.1104047108 [PubMed: 21646524]

Anderson BA, Laurent PA, Yantis S. Reward predictions bias attentional selection. Front Hum Neurosci. 2013; 7:262.doi: 10.3389/fnhum.2013.00262 [PubMed: 23781185]

Anderson BA, Laurent PA, Yantis S. Value-driven attentional priority signals in human basal ganglia and visual cortex. Brain Res. 2014; 1587:88-96. DOI: 10.1016/j.brainres.2014.08.062 [PubMed: 25171805]

Anderson BA, Leal SL, Hall MG, Yassa MA, Yantis S. The attribution of value-based attentional priority in individuals with depressive symptoms. Cogn Affect Behav Neurosci. 2014; 14(4):12211227. DOI: 10.3758/s13415-014-0301-z [PubMed: 24874421]

Anderson BA, Yantis S. Value-driven attentional and oculomotor capture during goal-directed, unconstrained viewing. Atten Percept Psychophys. 2012; 74(8):1644-1653. DOI: 10.3758/ s13414-012-0348-2 [PubMed: 22810561]

Anderson BA, Yantis S. Persistence of value-driven attentional capture. J Exp Psychol Hum Percept Perform. 2013; 39(1):6-9. DOI: 10.1037/a0030860 [PubMed: 23181684]

Barkley RA. Behavioral inhibition, sustained attention, and executive functions: constructing a unifying theory of ADHD. Psychol Bull. 1997; 121(1):65-94. [PubMed: 9000892]

Benjamini Y, Hochberg Y. Controlling the False Discovery Rate: A Practical and Powerful Approach to Multiple Testing. Journal of the Royal Statistical Society. Series B (Methodological). 1995; 57(1):289-300.

Bubnik MG, Hawk LW, Pelham WE, Waxmonsky JG, Rosch KS. Reinforcement Enhances Vigilance Among Children With ADHD: Comparisons to Typically Developing Children and to the Effects of Methylphenidate. J Abnorm Child Psychol. 2015; 43(1):149-161. DOI: 10.1007/ s10802-014-9891-8 [PubMed: 24931776]

Conners CK. Conners' Rating Scales - Revised North Tonawanda, New York: Mutli-Health Systems, Inc.; 1997

Conners CK. Conners 3 North Tonawanda, NY: Multi-Health Systems, Inc.; 2008

Della Libera C, Chelazzi L. Visual selective attention and the effects of monetary rewards. Psychol Sci. 2006; 17(3):222-227. DOI: 10.1111/j.1467-9280.2006.01689.x [PubMed: 16507062]

Della Libera C, Chelazzi L. Learning to attend and to ignore is a matter of gains and losses. Psychol Sci. 2009; 20(6):778-784. DOI: 10.1111/j.1467-9280.2009.02360.x [PubMed: 19422618]

DuPaul GJ, , Power TJ, , Anastopoulos AD, , Reid R. ADHD Rating Scale_IV New York, NY: Guilford Press; 1998

Engelmann JB, Pessoa L. Motivation sharpens exogenous spatial attention. Emotion. 2007; 7(3):668674. DOI: 10.1037/1528-3542.7.3.668 [PubMed: 17683222]

Engle RW, Tuholski SW, Laughlin JE, Conway AR. Working memory, short-term memory, and general fluid intelligence: a latent-variable approach. J Exp Psychol Gen. 1999; 128(3):309-331. [PubMed: 10513398]

Epstein JN, Brinkman WB, Froehlich T, Langberg JM, Narad ME, Antonini TN, Altaye M. Effects of stimulant medication, incentives, and event rate on reaction time variability in children with ADHD. Neuropsychopharmacology. 2011; 36(5):1060-1072. DOI: 10.1038/npp.2010.243 [PubMed: 21248722]

Faul F, Erdfelder E, Lang AG, Buchner A. G*Power 3: a flexible statistical power analysis program for the social, behavioral, and biomedical sciences. Behav Res Methods. 2007; 39(2):175-191. [PubMed: 17695343]

Folk CL, Leber AB, Egeth HE. Made you blink! Contingent attentional capture produces a spatial blink. Perception \& Psychophysics. 2002; 64(5):741-753. DOI: 10.3758/bf03194741 [PubMed: 12201333] 
Folk CL, Remington RW, Johnston JC. Involuntary covert orienting is contingent on attentional control settings. Journal of Experimental Psychology: Human Perception and Performance. 1992; 18(4): 1030-1044. DOI: 10.1037/0096-1523.18.4.1030 [PubMed: 1431742]

Fosco WD, Hawk LW Jr, Rosch KS, Bubnik MG. Evaluating cognitive and motivational accounts of greater reinforcement effects among children with attention-deficit/hyperactivity disorder. Behavioral and Brain Functions. 2015; 11(1):20.doi: 10.1186/s12993-015-0065-9 [PubMed: 25926127]

Fukuda K, Vogel EK. Individual differences in recovery time from attentional capture. Psychol Sci. 2011; 22(3):361-368. DOI: 10.1177/0956797611398493 [PubMed: 21310945]

Hazell PL, Carr VJ, Lewin TJ, Dewis SAM, Heathcote DM, Brucki BM. Effortful and automatic information processing in boys with ADHD and specific learning disorders. Journal of Child Psychology and Psychiatry. 1999; 40(2):275-286. DOI: 10.1111/1469-7610.00441 [PubMed: 10188710]

Hickey C, Chelazzi L, Theeuwes J. Reward changes salience in human vision via the anterior cingulate. J Neurosci. 2010a; 30(33):11096-11103. DOI: 10.1523/JNEUROSCI.1026-10.2010 [PubMed: 20720117]

Hickey C, Chelazzi L, Theeuwes J. Reward guides vision when it's your thing: trait reward-seeking in reward-mediated visual priming. PLoS One. 2010b; 5(11):e14087.doi: 10.1371/journal.pone. 0014087 [PubMed: 21124893]

Hollingshead AB. Four factor index of social status New Haven, CT: Yale University, Department of Sociology; 1975

Kaufman J, , Birmaher B, , Axelson D, , Perepletchikova F, , Brent D, , Ryan N. Kiddie Schedule for Affective Disorders and Schizophrenia for School-Aged Children - Lifetime Version (KiddieSADS-PL 2013 Working Draft) Pittsburgh Pennsylvania: Western Psychiatric Institute and Clinic and Yale University; 2013

Kiss M, Driver J, Eimer M. Reward priority of visual target singletons modulates event-related potential signatures of attentional selection. Psychological Science. 2009; 20(2):245-251. DOI: 10.1111/j.1467-9280.2009.02281.x [PubMed: 19175756]

Kristjansson A, Sigurjonsdottir O, Driver J. Fortune and reversals of fortune in visual search: Reward contingencies for pop-out targets affect search efficiency and target repetition effects. Attention, Perception, \& Psychophysics. 2010; 72(5):1229-1236. DOI: 10.3758/APP.72.5.1229

Laurent PA, Hall MG, Anderson BA, Yantis S. Valuable Orientations Capture Attention. Vis cogn. 2015; 23(1-2):133-146. DOI: 10.1080/13506285.2014.965242 [PubMed: 26146477]

Le Pelley ME, Pearson D, Griffiths O, Beesley T. When goals conflict with values: counterproductive attentional and oculomotor capture by reward-related stimuli. J Exp Psychol Gen. 2015; 144(1): 158-171. DOI: 10.1037/xge0000037 [PubMed: 25420117]

Luciana M. Practitioner review: computerized assessment of neuropsychological function in children: clinical and research applications of the Cambridge Neuropsychological Testing Automated Battery (CANTAB). Journal of Child Psychology and Psychiatry. 2003; 44(5):649-663. [PubMed: 12831110]

Luman M, Oosterlaan J, Sergeant JA. The impact of reinforcement contingencies on AD/HD: a review and theoretical appraisal. Clin Psychol Rev. 2005; 25(2):183-213. DOI: 10.1016/j.cpr.2004.11.001 [PubMed: 15642646]

Luman M, Tripp G, Scheres A. Identifying the neurobiology of altered reinforcement sensitivity in ADHD: a review and research agenda. Neurosci Biobehav Rev. 2010; 34(5):744-754. DOI: 10.1016/j.neubiorev.2009.11.021 [PubMed: 19944715]

Martinussen R, Hayden J, Hogg-Johnson S, Tannock R. A meta-analysis of working memory impairments in children with attention-deficit/hyperactivity disorder. J Am Acad Child Adolesc Psychiatry. 2005; 44(4):377-384. DOI: 10.1097/01.chi.0000153228.72591.73 [PubMed: 15782085]

Mason DJ, Humphreys GW, Kent L. Visual search, singleton capture, and the control of attentional set in ADHD. Cogn Neuropsychol. 2004; 21(6):661-687. DOI: 10.1080/02643290342000267 [PubMed: 21038227] 
Mason DJ, Humphreys GW, Kent LS. Exploring selective attention in ADHD: visual search through space and time. Journal of Child Psychology and Psychiatry. 2003; 44(8):1158-1176. DOI: 10.1111/1469-7610.00204 [PubMed: 14626457]

Myerson J, Green L, Warusawitharana M. Area under the curve as a measure of discounting. J Exp Anal Behav. 2001; 76(2):235-243. DOI: 10.1901/jeab.2001.76-235 [PubMed: 11599641]

Navalpakkam V, Koch C, Perona P. Homo economicus in visual search. Journal of Vision. 2009; 9(1): 31-16. 31-16. DOI: 10.1167/9.1.31

Patros CH, Alderson RM, Kasper LJ, Tarle SJ, Lea SE, Hudec KL. Choice-impulsivity in children and adolescents with attention-deficit/hyperactivity disorder (ADHD): A meta-analytic review. Clinical Psychology Review. 2016; 43:162-174. DOI: 10.1016/j.cpr.2015.11.001 [PubMed: 26602954]

Peck CJ, Jangraw DC, Suzuki M, Efem R, Gottlieb J. Reward modulates attention independently of action value in posterior parietal cortex. J Neurosci. 2009; 29(36):11182-11191. DOI: 10.1523/ JNEUROSCI.1929-09.2009 [PubMed: 19741125]

Pessoa L, Engelmann JB. Embedding reward signals into perception and cognition. Front Neurosci. 2010; 4doi: 10.3389/fnins.2010.00017

Plichta MM, Scheres A. Ventral-striatal responsiveness during reward anticipation in ADHD and its relation to trait impulsivity in the healthy population: a meta-analytic review of the fMRI literature. Neuroscience and Biobehavioral Reviews. 2014; 38:125-134. DOI: 10.1016/j.neubiorev. 2013.07.012 [PubMed: 23928090]

Raymond JE, O'Brien JL. Selective visual attention and motivation: the consequences of value learning in an attentional blink task. Psychol Sci. 2009; 20(8):981-988. DOI: 10.1111/j. 1467-9280.2009.02391.x [PubMed: 19549080]

Reich W, , Welner Z, , Herjanic B. Diagnostic Interview for Children and Adolescents-IV (DICA-IV) North Tonawanda, NY: Multi-Health Systems, Inc.; 1997

Roper ZJ, Vecera SP, Vaidya JG. Value-driven attentional capture in adolescence. Psychological Science. 2014; 25(11):1987-1993. DOI: 10.1177/0956797614545654 [PubMed: 25210012]

Rosch KS, Fosco WD, Pelham WE Jr, Waxmonsky JG, Bubnik MG, Hawk LW Jr. Reinforcement and Stimulant Medication Ameliorate Deficient Response Inhibition in Children with AttentionDeficit/Hyperactivity Disorder. Journal of Abnorm Child Psychology. 2016; 44(2):309-321. DOI: 10.1007/s10802-015-0031-x

Rosch KS, Hawk LW Jr. The effects of performance-based rewards on neurophysiological correlates of stimulus, error, and feedback processing in children with ADHD. Psychophysiology. 2013; 50(11): 1157-1173. DOI: 10.1111/psyp.12127 [PubMed: 24033316]

Rosch KS, Mostofsky SH. Increased Delay Discounting on a Novel Real-Time Task among Girls, but not Boys, with ADHD. Journal of the International Neuropsychological Society. 2016; 22(1):1223. DOI: 10.1017/S1355617715001071 [PubMed: 26549118]

Sagvolden T, Johansen EB, Aase H, Russell VA. A dynamic developmental theory of attention-deficit/ hyperactivity disorder (ADHD) predominantly hyperactive/impulsive and combined subtypes. Behavioral and Brain Sciences. 2005; 28(3):397-419. discussion 419-368. DOI: 10.1017/ S0140525X05000075 [PubMed: 16209748]

Sali AW, Anderson BA, Courtney SM. Information processing biases in the brain: Implications for decision-making and self-governance. Neuroethics. 2016; doi: 10.1007/s12152-016-9251-1

Sali AW, Anderson BA, Yantis S. The role of reward prediction in the control of attention. J Exp Psychol Hum Percept Perform. 2014; 40(4):1654-1664. DOI: 10.1037/a0037267 [PubMed: 24955700]

Seitz AR, Kim D, Watanabe T. Rewards evoke learning of unconsciously processed visual stimuli in adult humans. Neuron. 2009; 61(5):700-707. DOI: 10.1016/j.neuron.2009.01.016 [PubMed: 19285467]

Shiels K, Hawk LW, Lysczek CL, Tannock R, Pelham WE, Spencer SV, Waschbusch DA. The effects of incentives on visual-spatial working memory in children with attention-deficit/hyperactivity disorder. J Abnorm Child Psychol. 2008; 36(6):903-913. DOI: 10.1007/s10802-008-9221-0 [PubMed: 18288603]

Shuler MG, Bear MF. Reward timing in the primary visual cortex. Science. 2006; 311(5767):16061609. DOI: 10.1126/science.1123513 [PubMed: 16543459] 
Sonuga-Barke EJ. Psychological heterogeneity in AD/HD--a dual pathway model of behaviour and cognition. Behav Brain Res. 2002; 130(1-2):29-36. [PubMed: 11864715]

Sonuga-Barke EJ. The dual pathway model of AD/HD: an elaboration of neuro-developmental characteristics. Neurosci Biobehav Rev. 2003; 27(7):593-604. [PubMed: 14624804]

Sonuga-Barke EJ, Bitsakou P, Thompson M. Beyond the dual pathway model: evidence for the dissociation of timing, inhibitory, and delay-related impairments in attention-deficit/hyperactivity disorder. J Am Acad Child Adolesc Psychiatry. 2010; 49(4):345-355. [PubMed: 20410727]

Strand MT, Hawk LW Jr, Bubnik M, Shiels K, Pelham WE Jr, Waxmonsky JG. Improving working memory in children with attention-deficit/hyperactivity disorder: the separate and combined effects of incentives and stimulant medication. J Abnorm Child Psychol. 2012; 40(7):1193-1207. DOI: 10.1007/s10802-012-9627-6 [PubMed: 22477205]

Theeuwes J. Perceptual selectivity for color and form. Percept Psychophys. 1992; 51(6):599-606. [PubMed: 1620571]

Theeuwes J. Stimulus-driven capture and attentional set: selective search for color and visual abrupt onsets. J Exp Psychol Hum Percept Perform. 1994; 20(4):799-806. [PubMed: 8083635]

Theeuwes J. Top-down and bottom-up control of visual selection. Acta Psychologica. 2010; 135(2): 77-99. DOI: 10.1016/j.actpsy.2010.02.006 [PubMed: 20507828]

Van der Stigchel S, Rommelse NN, Deijen JB, Geldof CJ, Witlox J, Oosterlaan J, Theeuwes J. Oculomotor capture in ADHD. Cogn Neuropsychol. 2007; 24(5):535-549. DOI: 10.1080/02643290701523546 [PubMed: 18416506]

Visser SN, Danielson ML, Bitsko RH, Holbrook JR, Kogan MD, Ghandour RM, Blumberg SJ. Trends in the parent-report of health care provider-diagnosed and medicated attention-deficit/hyperactivity disorder: United States, 2003-2011. J Am Acad Child Adolesc Psychiatry. 2014; 53(1):34-46. e32. [PubMed: 24342384]

Wechsler DL. Wechsler Individual Achievement Test - Second Edition (WIAT-II) San Antonio, TX: The Psychological Corporation; 2002

Wechsler DL. Wechsler Intelligence Scale for Children - Fourth Edition (WISC-IV) San Antonio, TX: The Psychological Corporation; 2003

Wechsler DL. Wechsler Intelligence Scale for Children - Fifth Edition (WISC-V) The Psychological Corporation; 2014

Willcutt EG, Nigg JT, Pennington BF, Solanto MV, Rohde LA, Tannock R, Lahey BB. Validity of DSM-IV Attention Deficit/Hyperactivity Disorder Symptom Dimensions and Subtypes. J Abnorm Psychol. 2012; doi: 10.1037/a0027347

Wilson VB, Mitchell SH, Musser ED, Schmitt CF, Nigg JT. Delay discounting of reward in ADHD: application in young children. Journal of Child Psychology and Psychiatry. 2011; 52(3):256-264. DOI: 10.1111/j.1469-7610.2010.02347.x [PubMed: 21083561]

Wolfe JM, Cave KR, Franzel SL. Guided search: An alternative to the feature integration model for visual search. Journal of Experimental Psychology: Human Perception and Performance. 1989; 15(3):419-433. DOI: 10.1037/0096-1523.15.3.419 [PubMed: 2527952]

Yantis S, Anderson BA, Wampler EK, Laurent PA. Reward and attentional control in visual search. Nebr Symp Motiv. 2012; 59:91-116. [PubMed: 23437631] 
a

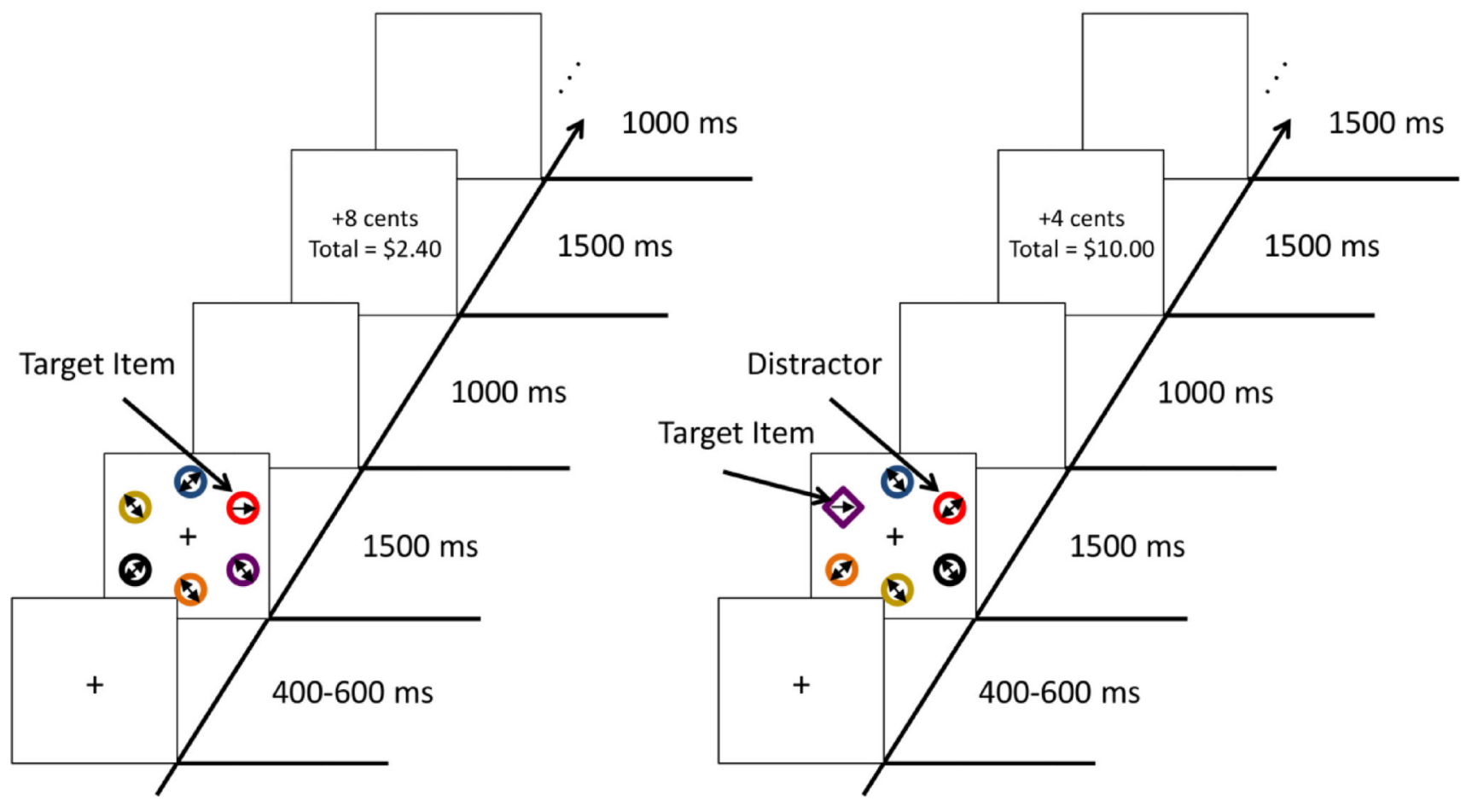

Figure 1.

(a) Training phase value-driven attentional capture task. (b) Test phase value-driven attentional capture task. 
a

\section{Training Phase}

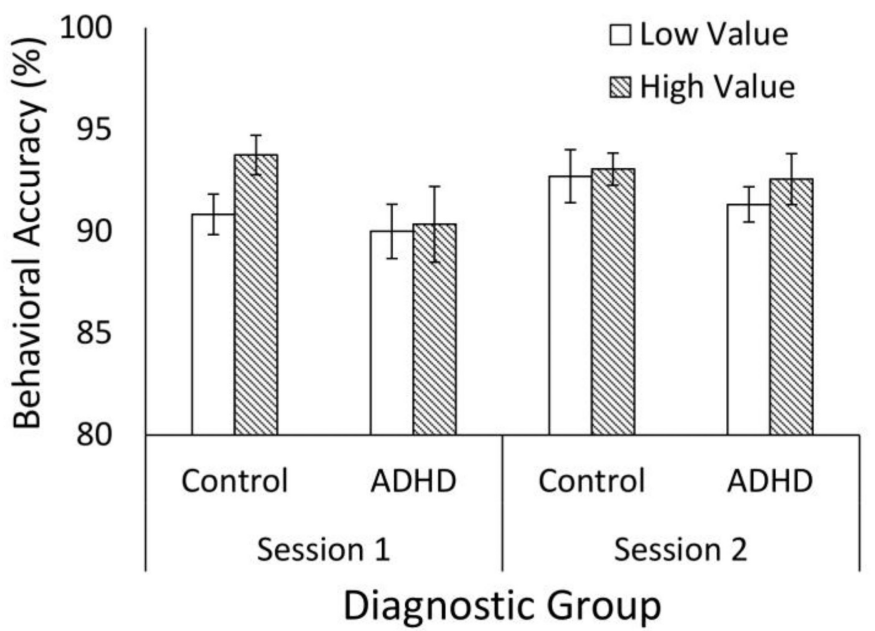

b

Figure 2.

Behavioral accuracies during (a) both training phase sessions and (b) the test phase of the VDAC task. Error bars denote 1 standard error of the mean above and below each mean. 


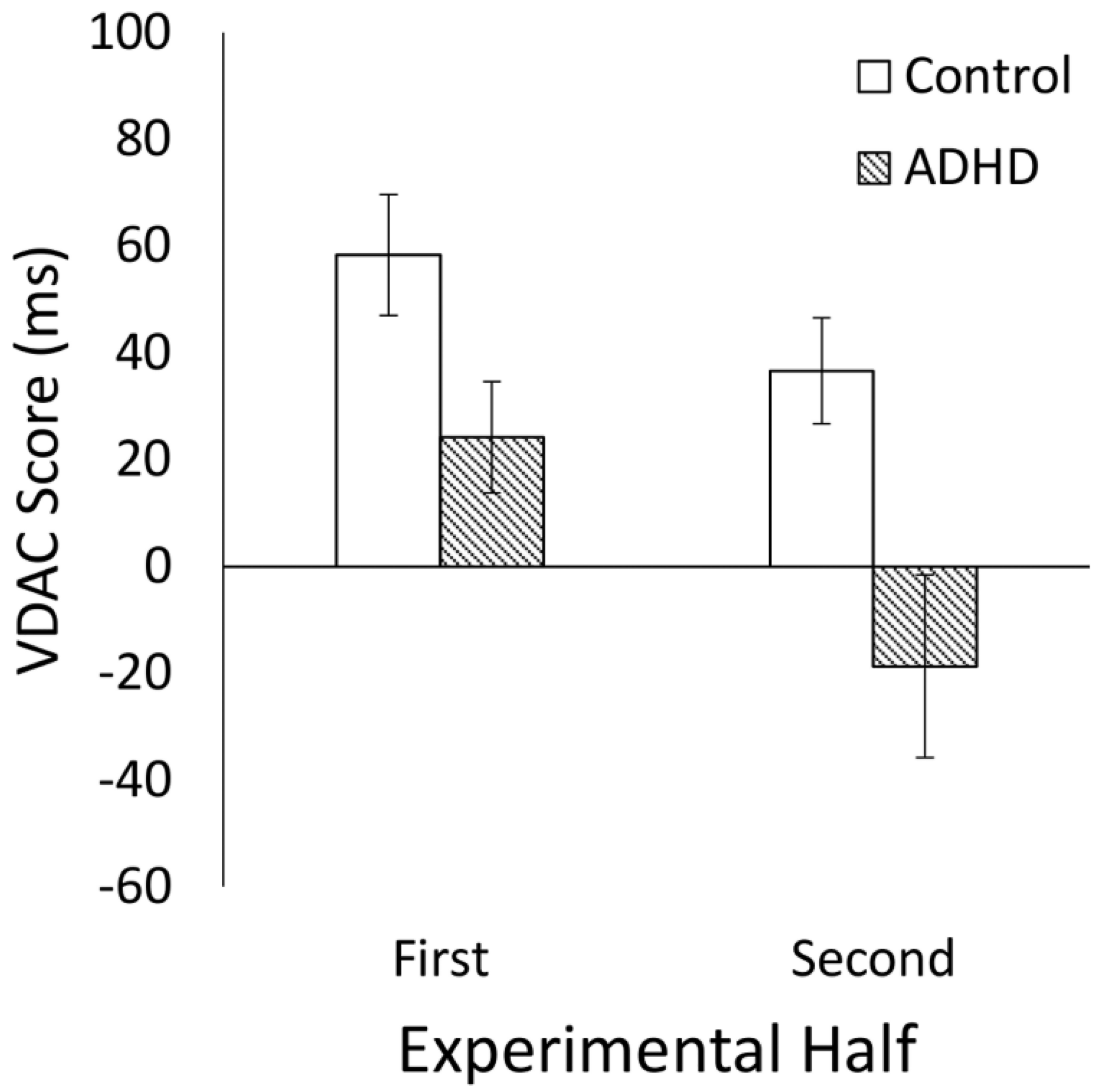

Figure 3.

Value-driven attentional capture scores (distractor present RT - distractor absent RT) as a function of diagnostic group over the course of the test phase. Error bars denote 1 standard error of the mean above and below each mean. 
a

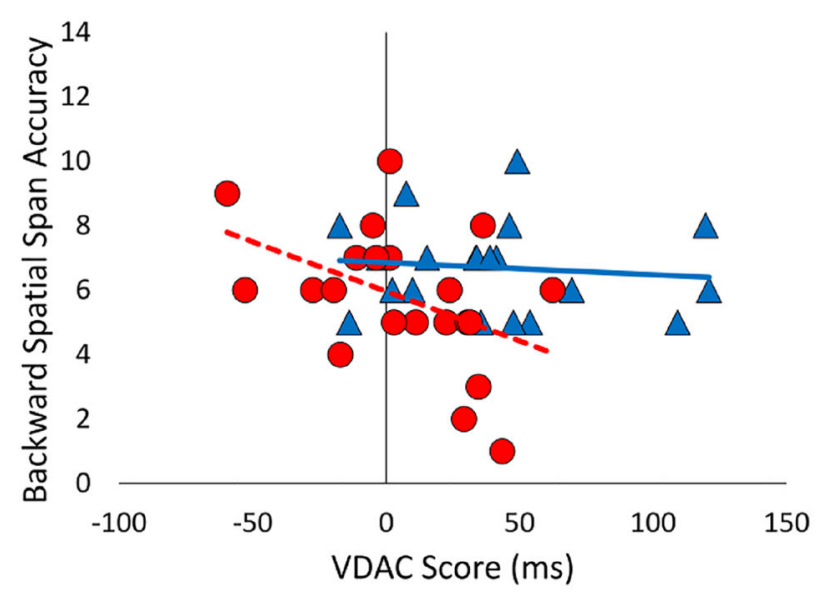

b

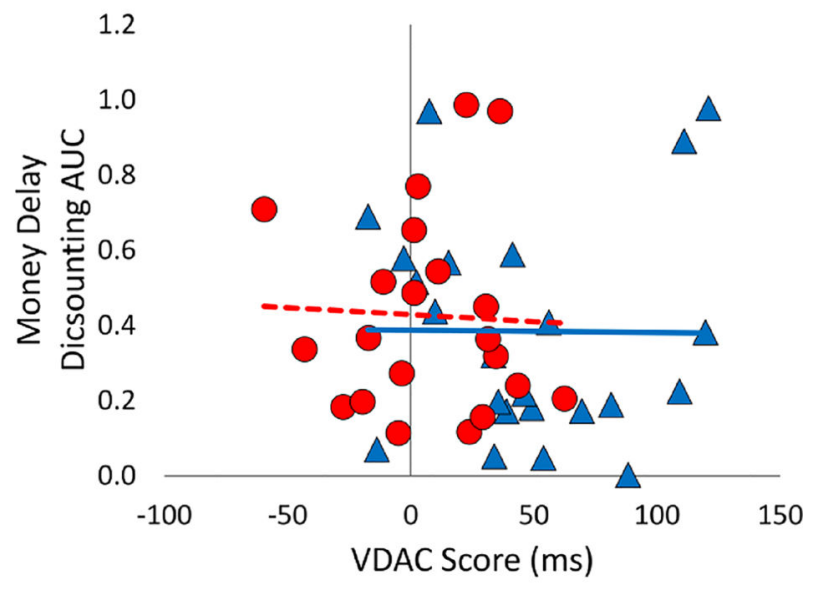

\section{$\rightarrow$ Control ....... ADHD}

Figure 4.

Correlations between value-driven attentional capture scores and (a) backward spatial span, (b) money delay discounting AUC. 


\section{Table 1}

Demographic and clinical characteristics of study participants.

\begin{tabular}{lccc}
\hline & $\begin{array}{c}\text { Control } \\
\boldsymbol{n}=\mathbf{2 4}\end{array}$ & $\begin{array}{c}\text { ADHD } \\
\boldsymbol{n}=\mathbf{2 4}\end{array}$ & Group Comparisons \\
\cline { 2 - 4 } & Mean (SD) & Mean (SD) & $\boldsymbol{p}$-value \\
\cline { 2 - 4 } Age (years) & $10.2(1.2)$ & $10.4(1.3)$ & .468 \\
Sex (boys: girls) & $13: 11$ & $17: 7$ & .233 \\
Ethnicity (\% caucasian) & $58 \%$ & $67 \%$ & .551 \\
SES & $52.6(9.6)$ & $54.3(10.0)$ & .555 \\
WISC FSIQ & $112.6(12.3)$ & $109.0(8.4)$ & .244 \\
WISC GAI & $112.7(13.5)$ & $111.2(9.3)$ & .666 \\
ADHD-RS Inatt Raw & $3.9(3.2)$ & $19.3(4.2)$ & $<.001$ \\
ADHD-RS HypImp Raw & $2.4(2.7)$ & $13.8(6.8)$ & $<.001$ \\
CPRS Inatt T & $45.4(7.1)$ & $76.6(9.2)$ & $<.001$ \\
CPRS HypImp T & $45.1(6.0)$ & $71.9(16.2)$ & $<.001$ \\
ADHD Subtype (Com:Inatt) & $\mathrm{n} / \mathrm{a}$ & $17: 7$ & $\mathrm{n} / \mathrm{a}$ \\
Comorbid ODD \% & $\mathrm{n} / \mathrm{a}$ & $38 \%$ & $\mathrm{n} / \mathrm{a}$ \\
Stimulant medication \% & $\mathrm{n} / \mathrm{a}$ & $42 \%$ & $\mathrm{n} / \mathrm{a}$
\end{tabular}

Notes: Control = typically developing control group; $\mathrm{ADHD}=$ attention-deficit/hyperactivity disorder group; SES = socioeconomic status from Hollingshead total score; WISC FSIQ = Wechsler Intelligence Scale for Children Fourth $(n=24)$ or Fifth $(n=24)$ Edition Full Scale Intelligence Quotient; WISC GAI = General Ability Index; ADHD-RS Inatt Raw = DuPaul Parent Inattentive raw score (range 0-27); ADHD-RS HypImp Raw = DuPaul Parent Hyperactive/Impulsive raw score (range 0-27); CPRS Inatt $\mathrm{T}=$ Conners Parent Rating Scale-Revised ( $\mathrm{n}=2$ ) or Conners-3 Parent $(\mathrm{n}=50)$ DSM Inattentive Index T-score; CPRS HypImp T = Conners DSM Hyperactive/Impulsive Index T-score; ADHD Subtype $($ Com: Inatt $)=$ ADHD Combined and Inattentive subtypes; ODD = Oppositional Defiant Disorder. 


\section{Table 2}

ANOVA results

\begin{tabular}{lcc}
\hline Effect & $\begin{array}{c}\boldsymbol{F} \text {-statistic (p- } \\
\text { value) }\end{array}$ & $\begin{array}{c}\text { Partial eta- } \\
\text { squared }\end{array}$ \\
\hline Training Phase Behavioral Accuracies & & \\
Main Effect of Session & $3.59(.065)$ & .072 \\
Main Effect of Target Value & $3.20(.08)$ & .065 \\
Main Effect of Diagnostic Group & $1.25(.269)$ & .026 \\
Diagnostic Group $\times$ Target Value & $0.38(.543)$ & .008 \\
Diagnostic Group $\times$ Session & $0.90(.349)$ & .019 \\
Session $\times$ Target Value & $0.76(.388)$ & .016 \\
Diagnostic Group $\times$ Session $\times$ Target Value & $3.29(.076)$ & .067 \\
Test Phase Behavioral Accuracies & & \\
Main Effect of Diagnostic Group & $5.33(.025)$ & .104 \\
Main Effect of Distractor Presence & $2.40(.128)$ & .050 \\
Diagnostic Group $\times$ Distractor Presence & $0.01(.944)$ & $<.001$ \\
Test Phase VDAC & & \\
Main Effect of Diagnostic Group & $14.97(<.001)$ & .246 \\
Main Effect of Experimental Half & $5.76(.021)$ & .111 \\
Diagnostic Group $\times$ Half & $0.62(.436)$ & .013 \\
&
\end{tabular}

